\section{Stemming ovarian cancer}

\section{By Lev Osherovich, Senior Writer}

A Boston team has identified ovarian cancer stem cells that may be responsible for the disease's high relapse rate. ${ }^{1}$ The group also discovered that a naturally occurring hormone called anti-Mullerian hormone can wipe out the stem cells, but recombinant production of it could prove challenging.

In recent years, oncologists have identified cancer stem cell markers in a number of solid tumors, but the precise identity of ovarian cancer stem cells has been a point of contention.

Now, a Massachusetts General Hospital (MGH) team led by Patricia Donahoe has identified a fraction of ovarian cancer cells with the molecular hallmarks of ovarian cancer stem cells.

Donahoe is professor of surgery and director of the pediatric surgical research laboratory at MGH.

The suspected cells are resistant to conventional chemotherapy but are highly sensitive to anti-Mullerian hormone (AMH). AMH suppresses growth of the female reproductive system during male embryonic development.

According to Donahoe, AMH works by arresting ovarian cancer stem cells during cell division, which eventually leads to apoptosis. She thus suspects that AMH could be used to prevent the recurrence of ovarian tumors.

$\mathrm{AMH}$ "is a natural substance found in the fetus that causes regression of the female reproductive organs," said Donahoe. "Our initial hypothesis many years ago was that if this agent caused regression of a whole organ system, it could also regress tumors derived from that organ system."

But until now, difficulties in making recombinant $\mathrm{AMH}$ and in identifying ovarian cancer stem cells have thwarted Donahoe's efforts to test her hypothesis.

\section{Sex cells}

In a previous study, Donahoe's team found a small subset of aggressive ovarian cancer cells that could not absorb a cell-staining dye and were highly sensitive to AMH. ${ }^{2}$ Because dye exclusion is a characteristic of cancer stem cells in other tissues, Donahoe suspected that these AMHsensitive cells might have other stem cell-like properties.

In the new study, Donahoe's team analyzed the surface markers and growth characteristics of a variety of cultured ovarian cancer lines and primary tumor cells isolated from patient ascites.

The team sorted tumor cells using a panel of 130 antibodies against surface markers and tested each fraction for its ability to form tumor colonies in cell culture.

Cells that bore a combination of three cell surface proteins-CD24, CD44 and epithelial cell adhesion molecule (EpCAM) - grew more aggressively in cell culture and mouse xenografts than cells lacking the three markers.

The team then analyzed the dye-excluding subset of cells from its previous study and found that the same three surface markers were highly expressed in those cells compared with the bulk of ovarian cancer cell lines.

Cells expressing CD24, CD44 and EpCAM were relatively resistant to conventional chemotherapeutics like doxorubicin compared with cells without the markers from the same tumor line. Conversely, growth of these marker-bearing cells was inhibited by AMH. The hormone had little effect on tumor cells lacking the markers.

Results were published in the Proceedings of the National Academy of Sciences.

\section{Triple threat}

Altogether, the findings help pin down the identity of ovarian cancer stem cells, which have been hard to define because of variability between different ovarian tumors.

Although CD24, CD44 and EpCAM have each been found in stem cells of other solid tumors, Donahoe's results suggest that cells that bear all three markers are the progenitors of ovarian tumors.

Donahoe's study "is adding to what we know about the potential characteristics of a cancer-initiating population," said Denise Connolly, assistant professor in the women's cancer program at Fox Chase Cancer Center. "They did a very extensive characterization of surface markers and came up with a panel of markers" for a distinct subpopulation of cells that appear to be tumor progenitors.

According to Susan Murphy, research assistant professor of obstetrics and gynecology at Duke University, other teams have identified stem cell-like ovarian tumor cells bearing CD44 but have turned up other surface markers that Donahoe's team did not find, including CD117 and keratin 17 (KRT17; CK17). ${ }^{3,4}$

Thus, said Murphy, it is not clear how Donahoe's cells relate to the putative ovarian cancer stem cells identified by other teams.

One possibility is that cells with the combination of three markers found by Donahoe could be one of several populations of ovarian cancer cells with stem cell-like properties.

"How do these cells relate to one another? Are there multiple ways to get an ovarian tumor?" Murphy asked.

To tackle these questions, Donahoe is collaborating with Connolly to transplant putative cancer stem cells found by her team and others into a new mouse model of ovarian cancer. ${ }^{5}$

Connolly's model uses mice genetically engineered to develop ovarian tumors rather than getting them from xenografts. In this model, it's possible to transplant individual tumor cells isolated from one mouse into another and test whether these cells grow up into the full spectrum of cell types found in a tumor. 


\section{ANALYSIS}

\section{TARGETS \& MECHANISMS}

"The classic property of stem cells is selfrenewal," said Connolly. "If a small number of these cells can initiate a new tumor that contains more of these cells plus the other tumor cell types," this would provide evidence of a bona fide stem cell identity.

\section{Recurring insight}

Whatever the answer to the preceding questions, Donahoe plans to begin mouse testing to see if using conventional chemotherapy to kill the bulk of tumor cells and AMH to wipe out ovarian cancer stem cells could prevent disease recurrence.

"We're very successful at treating and debulking ovarian tumors with chemotherapy and surgery, but 70\% of these tumors recur after 9-12 months," said Donahoe. "This tumor-initiating population might be responsible for this recurrence."

If Donahoe's cells can indeed reconstitute full tumors, the next step will be to treat mice with AMH plus chemotherapy and compare the results with those from animals receiving chemotherapy alone.

Donahoe noted that the hormone has no effect on tissues that lack an appropriate receptor and thus is likely to have low toxicity.

"The doses we anticipate using in cancer are actually below those secreted by the testes of baby boys, making it very attractive in terms of toxicity," said Donahoe.

Scaling up the manufacturing of AMH may be a challenge. Donahoe said she previously out-licensed a patent covering AMH as a cancer therapeutic to Biogen Idec Inc. According to Donahoe, the hormone proved hard to make recombinantly and Biogen Idec returned the IP to MGH.

If AMH itself doesn't work out as a therapeutic agent, Donahoe may have a plan B. Her team previously reported that SP600125, a small molecule research reagent, works in part as an agonist of anti-Mullerian hormone receptor type II (AMHR2). ${ }^{6}$ In the PNAS paper, her group reported that SP600125 inhibited the growth of the suspected ovarian stem cells, albeit at much higher doses than AMH.

SP600125 will need to undergo optimization to improve its affinity for AMHR2. Donahoe and her colleagues at MGH filed a patent on the use of SP600125 in ovarian cancer in 2008. The patent has not yet been out-licensed.

Meanwhile, Christopher Nicodemus, $\mathrm{CSO}$ and cofounder of cancer mAb company Advanced Immune Therapeutics Inc., thinks Donahoe's marker proteins themselves could be promising immunotherapeutic targets.

"If you've identified a population of the real stem cells, you can characterize their markers to open a strategy for targeted immunotherapy," he noted.

Osherovich, L. SciBX 3(43); doi:10.1038/scibx.2010.1283

Published online Nov. 4, 2010

\section{REFERENCES}

1. Wei, X. et al. Proc. Natl. Acad. Sci. USA; published online Oct. 15, 2010; doi:10.1073/pnas.1012667107

Contact: Patricia Donahoe, Massachusetts General Hospital, Boston, Mass.

e-mail: pdonahoe@partners.org

2. Szotek, P.P. et al. Proc. Natl. Acad. Sci. USA 103, 11154-11159 (2006)

3. Zhang, S. et al. Cancer Res. 68, 4311-4320 (2008)

4. Feng, D. et al. Oncol. Rep. 22, 1129-1134 (2009)

5. Quinn, B.A. et al. J. Ovarian Res. 3, 24 (2010)

6. Renlund, N. et al. Endocrinology 149, 108-115 (2008)

\section{COMPANIES AND INSTITUTIONS MENTIONED}

Advanced Immune Therapeutics Inc., Wellesley Hills, Mass.

Biogen Idec Inc. (NASDAQ:BIIB), Weston, Mass.

Duke University, Durham, N.C.

Fox Chase Cancer Center, Philadelphia, Pa.

Massachusetts General Hospital, Boston, Mass. 